\title{
Improving the Performance of Transparent PLEDs with LiF/Ag/ITO Cathode
}

\author{
Kou-Chen Liu, ${ }^{\mathrm{a}, \mathrm{z}}$ Chao-Wen Teng, ${ }^{\mathrm{a}}$ Yen-Hsun Lu, ${ }^{\mathrm{a}}$ Jiun-Haw Lee, ${ }^{\mathrm{b}}$ and \\ Lai-Cheng Chen ${ }^{\mathrm{c}}$
}

${ }^{a}$ Graduate Institute of Electro-optical Engineering, University of Chang Gung, Kwei-Shan Tao-Yuan 333

Taiwan

${ }^{b}$ Graduate Institute of Electro-optical Engineering and Department of Electrical Engineering, Taiwan

University, Taipei, Taiwan

${ }^{c}$ Delta Optoelectronics, Incorporated, Hsinchu 30076, Taiwan

\begin{abstract}
This work demonstrates the favorable performance of a transparent polymeric light-emitting diode (PLED) using an effective $\mathrm{LiF} / \mathrm{Ag}$ /indium tin oxide (ITO) cathode. An Ag layer can prevent the underlying emitting layer from damage by bombardment during ITO sputtering. Devices that have an Ag layer have a lower driving voltage and lower leakage current than those without. $\mathrm{X}$-ray photoelectron spectroscopy data show that $\mathrm{Ag}$ increases the concentration of $\mathrm{Li}$ in the polymer, enhancing its electron injection and transport capacities. The lower leakage current results from the fact that the work function of Ag is lower than that of ITO. The insertion of the Ag layer also enhances the optical characteristics by improving carrier balance and reducing sputtering damage.
\end{abstract}

(C) 2007 The Electrochemical Society. [DOI: 10.1149/1.2757116] All rights reserved.

Manuscript submitted May 3, 2007; revised manuscript received June 1, 2007. Available electronically July $19,2007$.

Organic light-emitting diodes (OLEDs) have attracted substantial attention because of their superior potential for use in flat-panel displays. ${ }^{1}$ The next generation of flat-panel displays must be able to reproduce high-quality moving images, be lightweight, and consume little power. Furthermore, transparent OLEDs are expected to be useful in high-resolution full-color displays, as well as in helmetmounted, windshield-mounted, or other "head-up" display applications. ${ }^{2}$ The double-transparent electrode, such as indium tin oxide (ITO), is applied to achieve this objective. Although various methods are available for depositing ITO films, ${ }^{3-5}$ sputtering deposition provides the benefits of high deposition rate and operating stability. ${ }^{6}$ Furthermore, some investigations have demonstrated that using the sputtering process to deposit a contact electrode offers better adhesion than conventional thermal evaporation, facilitating a decline in the operating voltage, especially when an effective cathode such as $\mathrm{Mg} / \mathrm{Ag}^{7}$ is adopted. However, at high operating voltages the fact that the resistivity of ITO is higher than that of metal is a critical issue as it may be detrimental in microdisplay applications. Inserting a thin metal, such as $\mathrm{Mg} / \mathrm{Ag}, \mathrm{Ca}$, or $\mathrm{Li}$ between ITO and the emitting layer has been considered to reduce the resistivity of ITO. ${ }^{8,9}$ The purpose of this metal is to reduce not only the resistivity but also the height of the electron injection barrier and usually a metal with a low work function is used. However, such metals are not well-suited in the operating environment, because they are highly chemically reactive and therefore oxidize during sputtering of the ITO cathode. ${ }^{10}$ Therefore, a new metal is sought to replace these low-work-function metals. Although the use of a noble metal may prevent oxidation, the fact that the high work function of the noble metal is likely to be associated with a large injection barrier should also be considered. Many efforts have been made to use widely adopted alkaline fluorides such as $\mathrm{LiF}, \mathrm{NaF}$, and $\mathrm{CsF},{ }^{11,12}$ and especially $\mathrm{LiF}$ to reduce the electron injection barrier height. Other researchers have also presented the benefits of using alkaline flourides such as chemical interaction, tunneling, and the interface dipole effect. ${ }^{13,14}$ Additionally, the insertion of the metal buffer layer is also expected to improve the light output. Ag metal exhibits relatively low optical absorption and has the highest conductivity of almost all metals. ${ }^{15}$ Smith et al. ${ }^{16}$ also noted that inserting an Ag buffer layer can efficiently guide the light output while providing protection for the underlying emitting layer during the ITO cathode deposition. During sputtering, the bombardment of the energetic particles generally induces the formation of defects and local trap sites, resulting

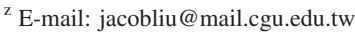

in the structural and electrical deterioration of organic films. Therefore, the thickness of the inserted metal is important and the metal must be able to prevent sputtering damage. According to Parthasarathy et al., ${ }^{17}$ the thickness of the insertion metal governs both the transparency and the damage due to bombardment. In this work, Ag insertion metal is employed for its superior stability, conductivity, and optical characteristics; the cathode configuration $\mathrm{LiF} / \mathrm{Ag} / \mathrm{ITO}$ is used to fabricate transparent PLEDs with low operating voltage, high electroluminescence (EL) performance, and favorable protection against sputtering damage.

The configuration of transparent PLED is ITO glass/poly(3,4ethylene dioxythiophene (PEDOT):poly(4-styrene sulfonate) (PSS)/ polyfluorene (PFO)/LiF/Ag/ITO. Because both electrodes are transparent, the light is emitted from the cathode and the anode. The ITO-glass substrates are cleaned by a treatment with $\mathrm{O}_{2}$ plasma. The polymer layers, PEDOT:PSS and PFO, are spin-coated and then baked in a nitrogen glove box containing less than $1 \mathrm{ppm}$ oxygen and moisture. The sample is then transferred to the thermal evaporation chamber to deposit $\mathrm{LiF}$ and $\mathrm{Ag}$, using an effusion cell and a heated resistor, respectively. The ITO cathode is deposited using a sputtering system. Finally, the devices are encapsulated in UVcuring epoxy resin. The transparency and resistivity of the $\mathrm{LiF} / \mathrm{Ag} / \mathrm{ITO}$ cathode are measured using a UV/visible/near-infrared (NIR) spectrometer (PerkinElmer Lamda 900) and a four-point probe (FPP 5000) to understand how the thickness of Ag influences these properties. The performance of the device is characterized using Keithly-2430 and Keithly-6485 measurement systems in an atmosphere of air at room temperature. The luminance is measured from both the anode and the cathode sides. X-ray photoelectron spectroscopy (XPS) measurements are made using a Perkin Elmer ESCA 5400 to elucidate the damage caused by bombardment during the sputtering of the ITO cathode.

Figure 1 demonstrates that inserting an $\mathrm{Ag}$ layer improves the current density-voltage $(J-V)$ characteristic. The turn-on voltage of the devices, defined as the bias at which the current density is $1 \mathrm{~mA} / \mathrm{cm}^{2}$, is 5.8, 5.5, and $4.8 \mathrm{~V}$ for devices with Ag layers of 1,3 , and $5 \mathrm{~nm}$ thickness; the corresponding voltage reduction percentages are 10, 15, and $26 \%$ from the voltage of the device with the $\mathrm{LiF} / \mathrm{ITO}$ cathode. Such a voltage reduction is associated with $(i)$ lower resistance of the Ag/ITO electrode, (ii) better injection from the cathode to the PFO, (iii) better electron transport capability in the PFO emitting layer [electromodulation of luminescence (EML)], and (iv) lower ion bombardment. Because the conductivity of $\mathrm{Ag}$ is two orders of magnitude higher than that of ITO, the resistivity of the electrode is substantially reduced when a thin Ag layer is introduced into the ITO thin film. ${ }^{15}$ The inset in Fig. 1 plots the resistiv- 


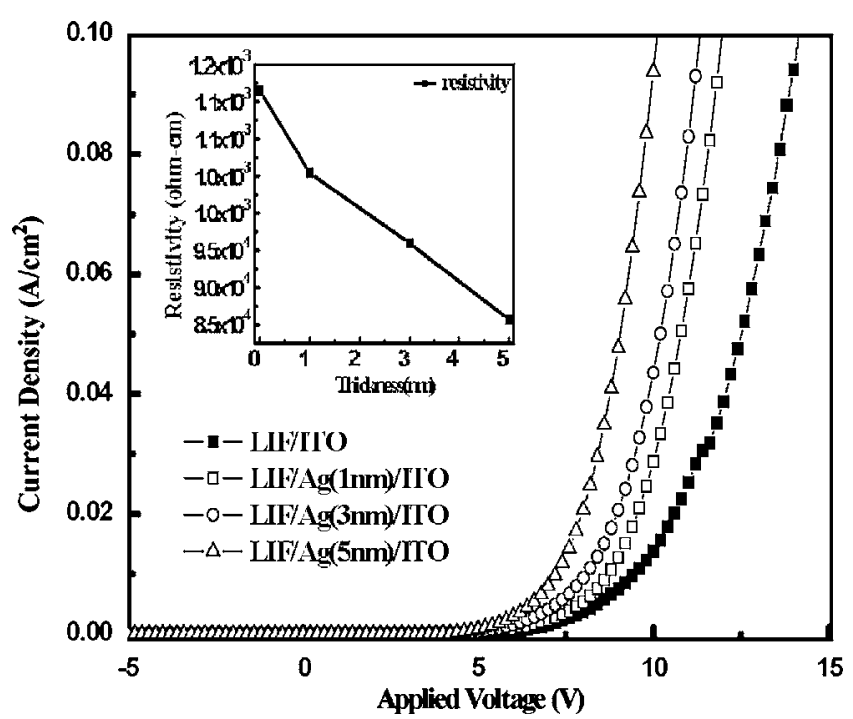

Figure 1. Current-voltage characteristic of transparent PLEDs with different thicknesses of Ag. The insertion is the resistivity of samples structure of $\mathrm{Si} / \mathrm{SiO}_{2} / \mathrm{Ag} / \mathrm{ITO}$.

ity of the $\mathrm{LiF} / \mathrm{Ag} / \mathrm{ITO}$ layer on the $\mathrm{Si} / \mathrm{SiO}_{2}$ wafer, which declines monotonically as the Ag thickness increases from 1-3 to $5 \mathrm{~nm}$. Kido et al. reported that the incorporation of $\mathrm{Li}$ into the organic materials can significantly improve the injection barrier from the cathode to the electron transport layer (ETL) and the electron transport characteristics. ${ }^{18}$ Although the $\mathrm{Li}$ ion is believed to be released from the $\mathrm{LiF}$ layer, numerous works have reported that $\mathrm{Ag}$ does not react with $\mathrm{LiF}$, because more heat is produced between $\mathrm{LiF}$ and $\mathrm{Ag}$ than by Al. However, the sputtering process of ITO deposition may provide the energy to overcome the reaction enthalpy. The sputtering process transforms kinetic energy to heat, which can activate the $\mathrm{LiF} / \mathrm{Ag}$ reaction. A thicker $\mathrm{Ag}$ layer provides more Ag to react with $\mathrm{LiF}$, liberating more $\mathrm{Li}$ to dope the emitting layer, as verified by XPS data. This reaction is similar to the LiF/Al reaction which was presented in previous works. ${ }^{13,14}$ Table I also shows that the concentration of $\mathrm{Li}$ in the EML increases with Ag thickness, providing direct evidence of the Li doping result. Additionally, inserting an Ag layer improves not only the electrical characteristics but also the optical properties, which are discussed later. The Ag layer is an effective buffer layer that prevents the formation of quench centers by sputtering damage and prevents exciton quenching. XPS measurements were made to characterize the phenomenon penetration into the emitting layer during sputtering ITO deposition. The compositions of ITO which include indium (In), tin (Sn), and oxygen (O) decreased sharply when a $1 \mathrm{~nm}$ thick Ag layer was inserted and continued to fall as the thickness of $\mathrm{Ag}$ was increased, as presented in Table I. This result indicates that the Ag film sufficiently prevents the impingement by high-kinetic-energy particles of In, Sn, and O. It also demonstrates that even a $1 \mathrm{~nm}$ thick layer used as the buffer layer can sufficiently suppress the sputtering bombardment into the

\section{Table I. The elements distribution of samples with different Ag} thicknesses.

Sample structure

The element in PFO

\begin{tabular}{lrcccr}
\cline { 5 - 6 } PFO/LiF/Ag/ITO & In $(\%)$ & Sn $(\%)$ & O $(\%)$ & Li $(\%)$ & F (\%) \\
\hline Ag $(0 \mathrm{~nm})$ & 14.27 & 1.01 & 3.6 & 3.59 & 8.77 \\
Ag $(1 \mathrm{~nm})$ & 8.33 & 0.42 & 2.29 & 4.8 & 14.54 \\
Ag $(3 \mathrm{~nm})$ & 4.17 & 0.27 & 2.1 & 5.6 & 12.24 \\
Ag $(5 \mathrm{~nm})$ & 2.49 & 0.11 & 1.4 & 8.03 & 10.06
\end{tabular}

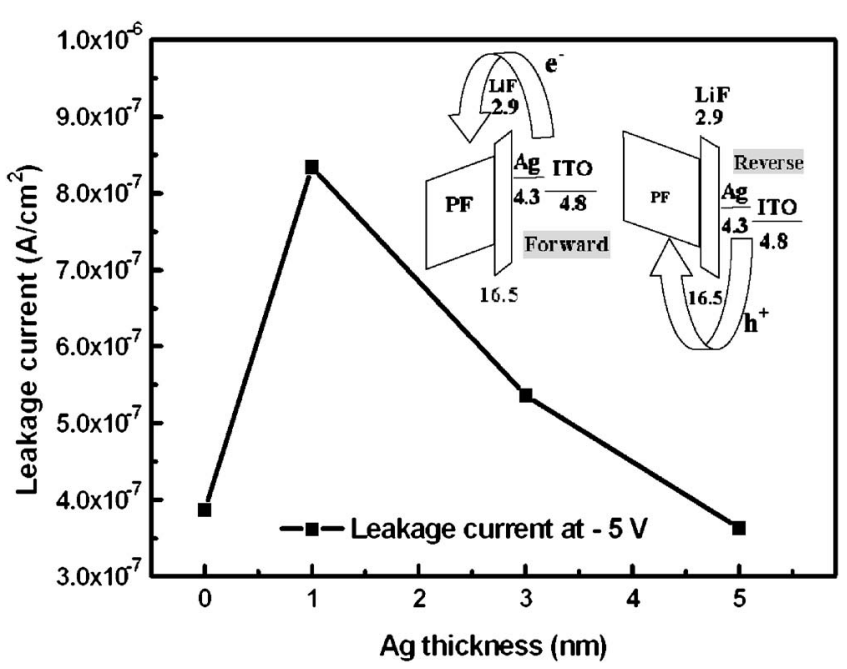

Figure 2. Leakage current variation with different Ag thicknesses.

emitting layer. For these two reasons, the devices with Ag layers have a higher current efficiency than those without. Figure 2 plots the leakage current density at $-5 \mathrm{~V}$ for various $\mathrm{Ag}$ thicknesses. The insertion of an $\mathrm{Ag}$ layer with a thickness of $1 \mathrm{~nm}$ increases the leakage current to double that in the LiF/ITO case. The leakage current decreases rapidly as the Ag thickness increases. When the $\mathrm{Ag}$ thickness is $5 \mathrm{~nm}$, the leakage current is even lower than the $\mathrm{LiF} / \mathrm{ITO}$ case. This work proposes a possible mechanism of this behavior. When the Ag layer is thin, 1 or $3 \mathrm{~nm}$, the ITO sputtering process oxidizes the $\mathrm{Ag}$ to form $\operatorname{Ag} x \mathrm{O}$, which has a high work function. ${ }^{15}$ Under reverse bias, the holes can be injected through these $\operatorname{Ag} x \mathrm{O}$ sites into the PFO, explaining the first increase in the leakage current when a thin Ag layer of 1 or $3 \mathrm{~nm}$ is inserted between the LiF and ITO. As the Ag thickness increases, the leakage current declines because the $\mathrm{Ag} / \mathrm{Ag} x \mathrm{O}$ ratio increases. When the $\mathrm{Ag}$ film is $5 \mathrm{~nm}$, the leakage current is even lower than that of ITO, because the lower work function of $\mathrm{Ag}$ is responsible for a higher barrier to the hole current under reverse bias, as presented in the inset in Fig. 2. Moreover, the presence of $\mathrm{LiF}$ also contributed to a high barrier to the hole current at reverse bias. According to our earlier investigation (not shown here), the device with an ITO cathode has a higher leakage current of $3.4 \times 10^{-6} \mathrm{~A} / \mathrm{cm}^{2}$. Figure 3 displays the total brightness, obtained by summing the top and bottom outputs of the transparent PLEDs. The brightness of devices with Ag buffer layers markedly exceeds that of those without. This result can be explained by the shift in the dipole recombination due to the lower resistivity of the cathode and the lower sputtering damage. The resistivity of the cathode is reduced when an $\mathrm{Ag}$ layer is inserted, strengthening the electrical field across PFO at a fixed voltage. Hence, the dipole recombination deposition shifts toward the HTL/EML interface. The dipole recombination region shifts far from the cathode, preventing electrode quenching and Li-diffusion quenching, which have been discussed elsewhere. ${ }^{19}$ Although devices with Ag layers all perform similarly and exhibit high luminance, the top/bottom emitting luminance ratio varies significantly with the Ag thickness in the devices, as shown in Fig. 4. The top/ bottom ratio initially increases when an Ag layer is inserted and then declines as the $\mathrm{Ag}$ thickness increases. As stated above, the incorporation of $\mathrm{Ag}$ shifts the recombination zone toward the HTL/EML interface and reduces the quenching effect at the cathode (top side). Therefore, the top emission from devices with Ag thickness of $1 \mathrm{~nm}$ exceeds that from $\mathrm{LiF} / \mathrm{ITO}$. The total emission of the OLED with $\mathrm{Ag}$ thickness of $1 \mathrm{~nm}$ is double that without $\mathrm{Ag}$ (Fig. 3), but the top/bottom ratio is only 60\% higher (Fig. 4), because the Ag film reflects some of the top-emitting photons and contributes to the bottom emission. According to Han et al., ${ }^{20}$ increasing the metal thick- 


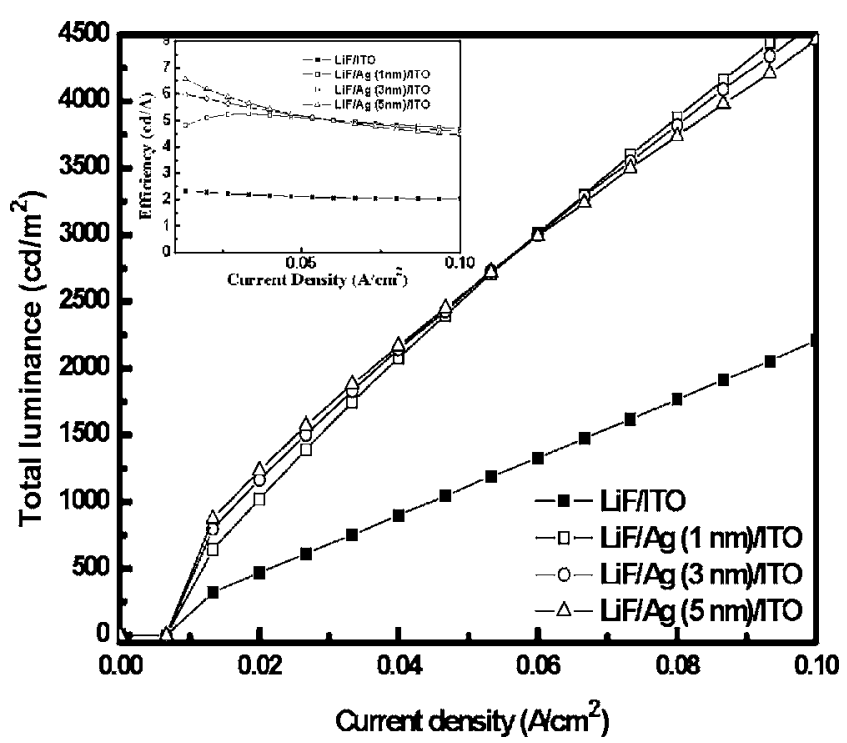

Figure 3. Total luminance (the sum of light from top and bottom) of devices with different Ag thicknesses. (Inset) Current efficiency vs current density.

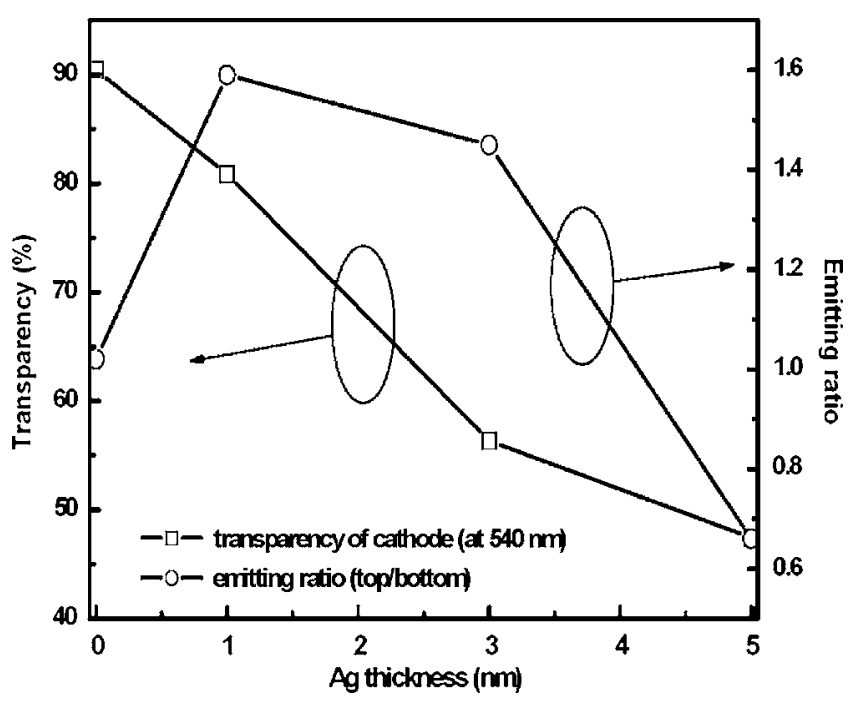

Figure 4. Transparency (at $540 \mathrm{~nm}$ ) of cathode and emitting ratio of device with different Ag thickness. ness drastically reduces resistivity and optical transparency. Figure 4 also demonstrates that the transparency of the cathode decreases as the Ag thickness increases. This result is consistent with Han's. Hence, the increase in reflectance associated with an increase in Ag thickness is conjectured to intensify the emission from the bottom side and the lower transparency is conjectured to reduce the light from the top side. Therefore, the emission ratio is reduced. However, further work is required to accurately detail the behavior of the internal waveguide.

This study demonstrates the favorable performance of transparent PLEDs with a thin Ag buffer layer. The presence of an Ag layer tunes the work function of the interface of the cathode not only to facilitate electron injection but also to suppress the leakage current. Additionally, inserting an Ag layer shelters the organic emitting layer from damage due to sputtering bombardment during the deposition from the top cathode, even when the Ag layer is thin.

\section{Acknowledgments}

The authors thank Delta Optoelectronics, Incorporated of Hsinchu, Taiwan, for supporting this research and Chung Shan Institute of Science and Technology (CSIST) for the facilitation of XPS measurements.

Chang Gung University assisted in meeting the publication costs of this article.

\section{References}

1. H. W. Choi, S. Y. Kim, K.-B. Kim, Y.-H. Tak, and J.-L. Lee, Appl. Phys. Lett., 86, 012104 (2005)

2. M. Pfeiffer, S. R. Forrest, X. Zhou, and K. Leo, Org. Electron., 4, 21 (2003).

3. H.-K.Kim, K.-S. Lee, M.-J. Geum, and K.-H. Kim, Electrochem. Solid-State Lett., 8, H103 (2005).

4. C. H. Chung, Y. W. Ko, Y. H. Kim, C. Y. Sohn, H. Y. Chu, and J. H. Lee, Appl. Phys. Lett., 86, 093504 (2005).

5. T. C. Gorjanc, D. Leong, C. Py, and D. Roth, Thin Solid Films, 413, 181 (2002).

6. T. Minami, H. Sonohara, T. Kakumu, and S. Takata, Thin Solid Films, 270, 37 (1995).

7. H. Suzuki and M. Hikita, Appl. Phys. Lett., 68, 2276 (1996).

8. G. Gu, V. Bulovi, P. E. Burrows, S. R. Forrest, and M. E. Thompson, Appl. Phys. Lett., 68, 2606 (1996).

9. L. S. Hung and J. Madathil, Thin Solid Films, 410, 101 (2002).

10. K. Tominaga, M. Chong, and Y. Shintani, J. Vac. Sci. Technol. A, 12, 1435 (1994).

11. J. Lee, Y. Park, D. Y. Kim, H. Y. Chu, H. Lee, and L. M. Do, Appl. Phys. Lett., 82 173 (2003).

12. S. L. Lai, M. Y. Chan, C. S. Lee, and S. T. Lee, J. Appl. Phys., 94, 7297 (2003).

13. M. G. Mason, C. W. Tang, L.-S. Hung, P. Raychaudhuri, J. Madathil, D. J. Giesen, L. Yan, Q. T. Le, Y. Gao, S.-T. Lee, et al., J. Appl. Phys., 89, 2756 (2001).

14. L. S. Hung, C. W. Tang, and M. G. Mason, Appl. Phys. Lett., 70, 152 (1997).

15. C. W. Chen, P. Y. Hsieh, H. H. Chiang, C. L. Lin, H. M. Wu, and C. C. Wu, Appl. Phys. Lett., 83, 5127 (2003)

16. L. H. Smith, J. A. E. Wasey, and W. L. Barnes, Appl. Phys. Lett., 84, 2986 (2004).

17. G. Parthasarathy, C. Adachi, P. E. Burrows, and S. R. Forrest, Appl. Phys. Lett. 76, 2128 (2000).

18. J. Kido and T. Matsumoto, Appl. Phys. Lett., 73, 2866 (1998)

19. L. S. Hung, L. S. Liao, C. S. Lee, and S. T. Lee, J. Appl. Phys., 86, 4607 (1999).

20. S. Han, D. Grozea, C. Huang, and Z. H. Lu, J. Appl. Phys., 96, 709 (2004) 